seen even more dramatically in the single recovery test. Here, the control Ss recovered the approach response only slightly (only three of the nine Ss recovered), while recovery was substantial for Group L (seven of the nine Ss recovered), and was even greater for Group $H$ (again, seven of the nine Ss recovered). The intergroup differences in the recovery test are significant, with Group $\mathrm{H}$ differing reliably from Group C (Duncan's Range Test, $\mathrm{p}<.05$ ).

\section{DISCUSSION}

Although the mechanism of the observed effect is still unclear, the following hypothesis may be suggested. Let us assume that running activity is ordinarily not aversive, and is incompatible with fear. In the present experiment, however, running occurs under rather unusual conditions. The $S$ is given an avoidance test trial, is immediately forced to run, is given another test trial immediately following running, and so on, i.e., the trials and interpolated activity are massed. Under these conditions, an aversive "defensive reaction" (see Lynn, 1966) might result from the novel and intense running stimulation which immediately follows a noxious experience in the avoidance apparatus. The $S$ is then tested in the presence of the aftereffects of this defensive reaction.

The avoidance behavior displayed by the forced activity groups during the massed avoidance trials may represent a summation of the effects of two sources of aversiveness: (a) the electric shock and its accompanying conditioned emotional response, and (b) the aftereffects of a defensive reaction. This second source of aversiveness would be most intense very early in testing, at the time of the S's first exposure to shock, conflict, and the running activity apparatus. With successive exposure, the defensive reaction and its aftereffects would habituate. During the 12-h rest period, residual aversive aftereffects would dissipate, allowing the usual fear-incompatible properties of running activity to become evident.

It would be interesting to determine whether running or other types of activity would reduce fear which was produced and measured in other ways. The influence of activity on other emotional states might also be investigated. Frustrating events have been shown to produce increased response vigor, activity, and attack (Amsel, 1967; Gallup, 1965). What would be the reciprocal effect of these latter behaviors on frustration? Some data that relate tangentially to this sort of question have been presented very recently by Moyer (1968), who has preliminary evidence which indicates that aggression circuits may be desensitized by the expression of aggressive behavior.

\section{REFERENCES}

AMSEL, A. Partial reinforcement effects on vigor and persistence. In $K . W$. Spence and J. T. Spence (Eds.), The psychology of learning and motivation. Vol. 1. New York: Academic Press, 1967.

GALLUP, G. G. Aggression in rats as a function of frustrative nonreward in a straight alley.
Psychonomic Science, 1965, 3, 99-100.

LYNN, R. Attention, arousal, and the orientation reaction. New York: Pergamon Press, 1966.

MOYER, K. A preliminary model of aggressive behavior. Paper presented at December 1968 Meeting of the American Association for the Advancement of Science, Dallas, Texas.

\title{
Electrically Induced affective attack from the hypothalamus of the albino rat'
}

JAAK PANKSEPP and JAY TROWILL, University of Massachusetts, Amherst, Mass. 01002

Electrically induced mouse killing was elicited by stimulation of the anterior lateral hypothalamus. Rats learned to press a bar to escape current levels that yielded attack. Attack latencies increased as test chamber size increased. With repeated testing sessions the vigor and amount of aggression decreased and somtimes disappeared with no corresponding decrease in escape behavior.

Among the behavior patterns elicited by electrical stimulation of the hypothalamus in the rat are eating, drinking, hoarding, maternal behavior, and increased sexual activity. Despite the observation that rats often attack and kill mice [(muricide) Karli, 1956], no evidence of centrally induced attack has been reported for the rat. Stimulus-bound attack, however, has been studied extensively in the cat. It has been divided behaviorally into affective attack and stalking attack (Wasman \& Flynn, 1962) for which the more general motivational correlates are escape (Adams \& Flynn, 1966) and approach (Roberts \& Kiess, 1964), respectively. The present report describes the preliminary results of stimulus- bound affective attack elicited from the hypothalamus of the albino rat. METHOD AND RESULTS

Animals were initially screened for muricide by leaving one albino mouse in the home cage of each rat. Only rats which did not kill the mouse in a $24 \mathrm{~h}$ period were implanted with bipolar electrodes aimed for selected areas of the hypothalamus. Each pole of an electrode was $.23 \mathrm{~mm}$ in diam and insulated except for the cross-section of the tip. After surgery Ss were screened in a $45 \times 40 \times 44 \mathrm{~cm}$ Plexiglas observation box. As possible goal objects of motivated behavior a live mouse, a dead mouse, two wedges of wood, and several food pellets were placed in the box during screening. Brain stimulation (60-cycle ac) was automatically programmed to be on for $25 \mathrm{sec}$ and off for $50 \mathrm{sec}$. Current was systematically varied each cycle and the motivational concomitants of stimulation were recorded. Of a total of more than 80 rats tested in this manner, we have observed stimulus-bound attack in 10 animals. This report is primarily based on data from these Ss.

In rats with effective electrode placements the aggressive behavior was similar to what has been described as affective at tack in cats. Attack was usually undirected and normally occurred when the paths of the rat and mouse crossed. Directed attack was observed to occur, however, if the rat was 
oriented toward the mouse when the stimulation came on. The attack usually consisted of a lethal bite around the neck region of the mouse (although bites on the hindquarters also occurred). With stimulus offset, ongoing attack immediately ceased. Rats never ate the mice they killed nor did they attack dead mice. On several trials, though, rats were observed to bite into a mouse they had just killed. Other behaviors often elicited at current levels yielding attack were ripping at the wood block, chattering of teeth, and backing up Although the current intensities necessary to evoke attack ranged from $15.70 \mu \mathrm{A}$ (mean $37 \mu \mathrm{A}$ ) across animals, within any one animal attack could only be obtained across a limited range of current intensities (e.g., as low as $5 \mu \mathrm{A}$ ). Current levels above optimum generally yielded intense escape behavior.

To assess the motivational aspects of this behavior more systematically, attack latencies were measured under different current intensities and in different size test chambers. The affective valence of the stimulation was also studied in selfstimulation and escape tests.

For current-intensity/attack-latency measurements rats were tested in a $23 \times 20 \times 19 \mathrm{~cm}$ Plexiglas box. Three mice were placed in the chamber to increase the likelihood that the rat was oriented toward a mouse at all times. The latency between stimulus onset and attack was measured. A minute was allowed between successive trials. Four Ss were tested on 3 days (10 trials per day) at two current levels separated by $5 \mu \mathrm{A}$. After each trial the mouse that was attacked was replaced. Within the effective current range the higher current level produced significantly faster attack than the low current (mean high-current latency$2.9 \mathrm{sec}$, low $-4.9 \mathrm{sec} ; \mathrm{p}<.01$, sign test).

Stimulus-bound attackers could not be trained to self-stimulate at any current intensity. Stimulation of these loci was clearly aversive since Ss were easily trained to press a bar to turn off the stimulation. The escape response was rapid and could consistently be obtained at current levels below those necessary for consistent attack behavior. It is known, however, that electric shock to the feet can induce fighting between rats (Ulrich \& Azrin, 1962). To control for nonspecific aversion-induced attack, other rats that had electrodes yielding equivalent escape behavior were screened for attack. None of these rats attacked mice at any current levels despite the fact that they made repeated contacts with the mouse while running around the observation chamber.

To further demonstrate that the stimulusbound attack was a manifestation of rage or defense rather than directed aggression, latency to attack was measured in test chambers of various sizes. It was hypothesized that attack latency should increase with larger test areas if the attack reflected a defense-rage reaction, but would not change significantly if it reflected an active seeking out of the prey as is characteristic of the directed biting attack of muricide. With current automatically programmed, time to attack was recorded in test areas of 600 , 1200 , and $1800 \mathrm{sq} \mathrm{cm}$. In four animals studied in this manner, latency to attack was unequivocally related to the size of the box. Attack was significantly slower $(p<.01)$ in successively larger areas. This supports the conclusion that this type of attack did not reflect active seeking out of a prey.

Prolonged observations of these rats have revealed a striking increase in latency of attack or complete cessation of stimulusbound attack behavior across testing sessions. In some cases there have been decrements within a session and recovery the next session, while in other animals the decrement has persisted up to a month with no intervening testing. Increasing current level does not reinstate attack. This effect is probably not an artifact of changes in effective current or in changes of the neural tissue at the site of stimulation, since in these same Ss the stimulation still produced agitation and left bar-press escape unabated. Such data emphasize the importance of experiential factors in stimulus-bound behaviors elicited from the hypothalamus. Experiential factors are beginning to be recognized as important concomitants of stimulus-bound behaviors. Increasing latency of attack across trials has been reported in a cat (Nakao, 1958), and within-session decrements in stimulusbound sexual activity have been reported for rats (Vaughan \& Fisher, 1962). It has also recently been shown that stimulus-bound eaters will assume the behavior of stimulusbound drinkers if food is removed but access to water remains (Valenstein, Cox, \& Kakolowski, 1968).

\section{HISTOLOGY}

Histological localization of electrode sites has been completed in five animals. Fifty-micron-thick frozen sections of electrode tracts were stained with cresyl violet. Effective electrodes were consistently located in the anterior lateral hypothalamus in tissue above the optic tract and optic chiasma. Electrodes in the optic tract did not elicit aggression. The two most consistent stereotaxic localizations for attack were $\mathrm{A}: 6.4, \mathrm{~L}: 1.8, \mathrm{H}:-3.0$; and A: $6.8, \mathrm{~L}: 2.0, \mathrm{H}:-2.4$ (de Groot, 1959). ${ }^{2}$

\section{DISCUSSION}

It should be emphasized that the stimulus-bound attack observed in this research is not similar to the muricide observed in normal rats. While muricide has been shown to be an effective reward in a learning situation (Myer \& White, 1965), and seems behaviorally analogous to stalking attack in cats, the stimulation necessary to induce attack in the present situation was unequivocally aversive and is similar to what has been called affective attack in cats. This data thus extends the generality of this type of stimulus-bound behavior across species.

The experience that causes some rats to stop attacking mice in the present situation is not clear. Perhaps the rat learns that the mice are not an adequate threat. Such a factor may operate in shock-induced attack since preliminary work from our laboratory has shown that foot shock does not induce rats to attack a restrained mouse. This hypothesis is also suggested by the behavior of one animal we have studied which did not attack mice when stimulated in the hypothalamus but did attack a more formidable opponent such as another rat. Although some type of learning seems to modulate centrally elicited attack in rats, the crucial factors remain to be defined.

\section{REFERENCES}

ADAMS, D., \& FLYNN, H. P. Transfer of an escape response from tail shock to brain stimulated attack behavior. Journal of the Experimental Analysis of Behavior, 1966, 9, 401-408.

de GROOT, J. The rat forebrain in stereotaxic coordinates. Verh. Kon. Ned. Akad. Wet., B. Naturkunde, 1959, 52, 1-40.

KARLI, P. The Norway rat's killing response to the white mouse. Behaviour, 1956, 10, 81-103.

KING, M. B. \& HOEBEL, B. G. Killing elicited by brain stimulation in rats. Communications in Behavioral Biology, 1968, 2, 173-177.

MYER, J. S., \& WHITE, R. T. Aggressive motivation in the rat. Animal Behaviour, 1965, 13,430-433.

NAKAO, H. Emotional behavior produced by hypothalamic stimulation. American Journal of Physiology, 1958, 194, 411-418.

ROBERTS, W. W., \& KIESS, H. O. Motivational properties of hypothalamic aggression in cats. Journal of Comparative \& Physiological Psychology, 1964, 58, 187-193.

ULRICH, R. E., \& AZRIN, N. H. Reflexive fighting in response to aversive stimulation. Journal of the Experimental Analysis of Behavior, 1962, 5, 511-520.

VALENSTEIN, E. S., COX, V. C., \& KAKOLEWSKI, J. W. Modification of motivated behavior elicited by electrical stimulation of the hypothalamus. Science, 1968, 159, 1119-1121.

VAUGHN, E., \& FISHER, A. E. Male sexual behavior induced by electrical stimulation. Science, 1962, 137, 758-760.

WASMAN, M., \& FLYNN, J. P. Directed attack elicited from hypothalamus. Archives of Neurology, 1962, 6, 220-227.

$$
\text { NOTES }
$$

1. This work was partially supported by USPHS Grant No. MH 13155-02 awarded to J. A. Trowill. 2. After the completion of this research, King \& Hoebel (1968) published a report of stimulusbound aggression from a different hypothalamic perifornical location. 